\title{
Medida da força de preensão manual - validade e confiabilidade do dinamômetro saehan
}

\author{
Assessment of hand grip strength - validity and reliability of the saehan dynamometer
}

Maurício Moreira Reis ${ }^{1}$ Paula Maria Machado Arantes ${ }^{1}$

Estudo desenvolvido na FISA Faculdade Itabirana de Saúde da Fundação Comunitária de Ensino Superior de Itabira, Itabira, MG, Brasil.

${ }^{1}$ Professores do Curso de Fisioterapia da Faculdade Itabirana de Saúde - Itabira (MG), Brasil.

\section{ENDERECO PARA CORRESPONDENNCIA:}

Maurício Moreira Reis - Vila técnica do areão, 12 - Bairro areão - CEP: 35900-841 Itabira (MG), Brasil - E-mail: fisiomauricio@yahoo.com.br

APRESENTACÃO: set. 2010

ACEITO PARA PUBLICAÇÃO: fev. 2011

FONTE DE FINANCIAMENTO: nenhuma

CONFLITO DE INTERESSE: Nada a declarar
RESUMO: Existe uma grande variedade de instrumentos utilizados para a avaliação da força de preensão manual. Porém, não existem estudos demonstrando a validade e a confiabilidade da maioria destes instrumentos. O objetivo deste estudo foi avaliar a validade concorrente e a confiabilidade intraexaminador do dinamômetro Saehan hidráulico comparado-o com o dinamômetro Jamar hidráulico. Cem indivíduos sadios (50 homens e 50 mulheres), entre 20 e 50 anos de idade, sem alterações cognitivas, deficiências físicas, disfunções neuromusculares e ortopédicas e história de lesões nos membros superiores, foram testados com os dinamômetros Jamar e Saehan. A validade concorrente entre o dinamômetro Jamar e o dinamômetro Saehan foi excelente para os testes de força de preensão realizados com as mãos direita $(r=0,976)$ e esquerda $(r=0,986)$. A confiabilidade intra-examinador foi excelente tanto para o dinamômetro Jamar ( $r=0,985$ mãos direita e esquerda) quanto para o dinamômetro Saehan ( $r=0,981$ mão direita e $r=0,985$ mão esquerda). O dinamômetro Saehan é válido, confiável e comparável com o dinamômetro Jamar. Portanto, dados coletados com o dinamômetro Jamar são equivalentes aos dados coletados com o dinamômetro Saehan. Consequentemente, valores de força obtidos por testes com dinamômetro Saehan podem ser comparados com valores de referência de força de preensão que foram obtidos com o dinamômetro Jamar.

Descritores: dinamômetro; força de preensão manual; validade; confiabilidade.

ABSTRACT: There is a great variety of instruments available for evaluating hand grip strength. There is, however, a lack of studies showing the validity and reliability of most of these instruments. The purpose of this study was to examine the concurrent validity and test-retest reliability of the hydraulic Saehan dynamometer using the hydraulic Jamar dynamometer. One hundred healthy subjects ( 50 men and 50 women), between the ages of 20 and 50 years old, without cognitive impairment, physical disability, neuromuscular or orthopedic dysfunction, and history of upper extremity injuries were tested with the Jamar and Saehan dynamometers. The concurrent validity between the Jamar and the Saehan dynamometers was excellent for both the right hand $(r=0.976)$ and the left hand $(r=0.986)$. Test-retest reliability was excellent for both the Jamar ( $r=0.985$ right and left hands) and the Saehan $(r=0.981$ right hand and $r=0.985$ left hand). The Saehan dynamometer is valid, reliable and comparable to the Jamar dynamometer. Therefore, the Jamar and Saehan dynamometers measure handgrip strength equivalently. As a result, strength values obtained with the Saehan dynamometer can be compared with reference values which were collected with the Jamar dynamometer.

KeYwoRDs: dynamometer; handgrip strength; validity; reliability. 


\section{INTRODUCÃO}

Diante da atual exigência de se demonstrar a efetividade de procedimentos clínicos, é essencial que fisioterapeutas e terapeutas ocupacionais provem a validade e a confiabilidade dos seus instrumentos de avaliação ${ }^{1}$. A avaliação válida e confiável da força de preensão manual é um parâmetro importante para determinar a efetividade de várias estratégias terapêuticas ${ }^{2,3}$, definir metas de tratamento, avaliar a habilidade do paciente para retornar a atividades funcionais, além de colaborar para o desenvolvimento de pesquisas científicas ${ }^{4}$.

A mensuração da força de preensão manual, através do dinamômetro, consiste em procedimento simples ${ }^{5}$, objetivo, prático e de fácil utilização ${ }^{6}$. Recomendado pela Sociedade Americana de Terapeutas de Mão $\left(\right.$ SATM $^{7}$ e pela Sociedade Americana para a Cirurgia da Mão $(\mathrm{SACM})^{8}$, o dinamômetro Jamar tem sido considerado o instrumento mais aceito para avaliação da força de preensão manual ${ }^{9-11}$, com alguns estudos apresentando dados normativos ${ }^{5,12-15}$. Existem dois tipos de dinamômetro Jamar, o digital e o hidráulico ${ }^{16-20}$. A literatura recomenda o uso do modelo hidráulico para a avaliação da força de preensão manual ${ }^{21,22}$. Vários estudos já reportaram alta confiabilidade e/ou validade deste tipo de dinamômetro ${ }^{23-29}$ e, por isto, este modelo é considerado o "padrão ouro" ${ }^{27,29}$ e já foi utilizado por vários pesquisadores como um instrumento padrão para validar outros equipamentos de medida da força de preensão manual18,23,26-31.

Existe uma grande variedade de instrumentos disponíveis comercialmente para a avaliação da força de preensão manual, incluindo o dinamômetro Saehan hidráulico. Este instrumento assemelha-se estruturalmente e funcionalmente ao dinamômetro Jamar. Porém, não existem estudos demonstrando sua validade e confiabilidade. Segundo Fess ${ }^{17}$, a confiabilidade e a validade são essenciais para determinar a efetividade de um instrumento de avaliação. Confiabilidade é definida como a extensão na qual uma medida é consistente e livre de erros $^{32}$. Quando um instrumento é capaz de medir uma variável precisamente e consistentemente em momentos distintos, considera-se que este instrumento apresenta uma boa confiabilidade intra- examinador $^{32}$. Validade é definida como a extensão na qual o instrumento mede aquilo que se pretende medir ${ }^{32}$. Para ser válido, um determinado instrumento deve ser confiável ${ }^{17}$. A forma mais objetiva de validade é a validade concorrente. Esta indica que os resultados dos testes obtidos com um instrumento podem substituir os resultados obtidos de testes realizados com um instrumento "padrão ouro" ${ }^{32}$.

Alguns estudos têm utilizado o dinamômetro Saehan hidráulico para avaliar a força de preensão ${ }^{33,34}$. Entretanto, as propriedades psicométricas deste instrumento de medida ainda precisam ser estabelecidas. Assim, o presente estudo teve o objetivo de avaliar a validade concorrente e a confiabilidade intraexaminador do dinamômetro Saehan hidráulico comparado-o com o dinamômetro Jamar hidráulico. A importância desta pesquisa está na necessidade de se garantir que instrumentos de avaliação utilizados nas pesquisas científicas e na prática clínica sejam válidos e confiáveis. A determinação destas propriedades poderá ser útil aos clínicos e pesquisadores que desejarem utilizar este instrumento, na prática clínica ou em pesquisas que envolvam a mensuração da força de preensão manual, principalmente considerando a acessibilidade do dinamômetro Saehan devido ao seu menor custo em relação a outros dinamômetros. A avaliação da validade deste dinamômetro também poderá contribuir para que dados normativos de força de preensão manual coletados com o dinamômetro Jamar possam ser utilizados como referência para testes realizados com o dinamômetro Saehan.

\section{METODOLOGIA}

Participaram do estudo 100 indivíduos sadios (50 homens e 50 mulheres) na faixa etária entre 20 e 50 anos de idade (média \pm desvio padrão, 29,39 $\pm 6,36$ anos), que consentiram em participar através da assinatura do Termo de Consentimento aprovado pelo Comitê de Ética em Pesquisa. Indivíduos com qualquer alteração cognitiva, deficiências físicas, disfunções neuromusculares e ortopédicas e história de lesões nos membros superiores, que possam afetar a força de preensão manual, não foram incluídos no estudo.
O dinamômetro Jamar hidráulico (Sammons Preston Rolyan, 4, Sammons Court, Bolingbrook, IL, 60440) e o dinamômetro Saehan hidráulico (Saehan Corporation, 973, Yangdeok-Dong, Masan 630-728, Korea) foram utilizados para a mensuração da força de preensão manual de cada participante. Ambos os dinamômetros foram calibrados antes do início da coleta dos dados e suas alças reguladas na segunda posição. Esta posição, considerada padrão pela SATM, é recomendada pela literatura para a realização dos testes de força de preensão na prática clínica e na pesquisa ${ }^{7,21,22}$.

Os participantes foram divididos em um grupo de homens e mulheres pares e um grupo de homens e mulheres ímpares. A força de preensão manual direita e esquerda do grupo de homens e mulheres pares foi mensurada com o dinamômetro Jamar primeiro e depois com o dinamômetro Saehan. O grupo de homens e mulheres ímpares foi avaliado na sequência oposta (Tabela 1). A sequência alternada teve o objetivo de eliminar a influência de potenciais efeitos da fadiga muscular nos testes ${ }^{28}$.

Estudos demonstram que os resultados dos testes de força de preensão manual podem ser influenciados por variações na posição do corpo ${ }^{35-38}$. Assim, os testes foram realizados na posição recomendada pela SATM. Os participantes ficaram sentados confortavelmente em uma cadeira sem braços, com os pés apoiados no chão e quadril e joelho posicionados a aproximadamente 90 graus de flexão. $\mathrm{O}$ ombro do membro testado ficou aduzido e em rotação neutra, cotovelo em flexão de 90 graus, antebraço na posição neutra e punho entre 0 e 30 graus de extensão e entre 0 a 15 graus de adução. A mão do membro não testado repousou sobre a coxa do mesmo lado (Figura 1). Os participantes foram instruídos a manter o posicionamento durante os testes e corrigidos pelo examinador quando necessário.

Acessórios tais como relógios, pulseiras, anéis e braceletes foram removidos de ambos os membros superiores dos participantes antes do início dos testes. Todos os participantes foram avaliados individualmente e em local reservado pelo mesmo avaliador nos períodos da manhã e da tarde. De acordo com Figueiredo et 
al. ${ }^{4}$ a divergência dos resultados de vários estudos ${ }^{10,39}$ parece indicar que o teste de força de preensão em variados horários do dia não deve ser motivo de preocupação. Foi dada uma demonstração de como o teste deveria ser realizado para a familiarização com o equipamento e os participantes fizeram uma simulação do teste realizando força submáxima tendo, em seguida, um período de 1 minuto de descanso antes do início do teste oficial. Os participantes foram orientados a não olhar para o mostrador do dinamômetro para evitar qualquer retorno (feedback) visual. Nenhum comando verbal foi dado durante o teste e as instruções para sua execução foram padronizadas. Durante as instruções, o volume do comando verbal permaneceu constante para se evitar qualquer influência do mesmo na magnitude da contração muscular ${ }^{40}$.

O protocolo de testes foi dividido em duas sessões. Na primeira sessão os testes foram realizados inicialmente com a mão direita e depois com a mão esquerda de forma não alternada, ou seja, três testes foram feitos consecutivamente com a mão direita e depois três testes foram feitos consecutivamente com a mão esquerda. Os participantes foram instruídos a fazer uma contração máxima por 3 segundos em cada teste. Houve um período de descanso de 30 segundos entre cada teste e um período de descanso de 2 minutos entre os testes de cada mão. Este procedimento foi feito primeiramente com um dos dinamômetros e, após um período de descanso de 2 minutos, repetido com o outro dinamômetro. Um intervalo de 10 minutos foi dado e todo o procedimento realizado na primeira sessão foi repetido na segunda sessão.

Foi utilizada a média dos valores dos três testes de cada mão para a análise dos dados. Este procedimento, recomendado pela $\mathrm{SATM}^{7}$ e por Figueiredo et al. ${ }^{4}$, é considerada a forma mais confiável de se medir a força de preensão manual ${ }^{41}$.

A validade concorrente entre os dinamômetros Jamar e Saehan e a confiabilidade intra-examinador de ambos os dinamômetros foram calculadas através do coeficiente de correlação intraclasse $(\mathrm{CCl})$. Um CCl de 0.90 ou maior é considerado excelente; de 0,75 a 0,90 bom; 0,50 a 0,75 moderado; e menos que 0,50 é considerado pobre ${ }^{32}$.
RESULTADOS

Os valores médios da força de preensão, das mãos direita e esquerda, coletados com os dinamômetros Jamar e Saehan estão demonstrados na Tabela 2.

A validade concorrente entre os dinamômetros Jamar e Saehan foi excelente para os testes de força de preensão realizados com as mãos direita $(r=0.976) \mathrm{e}$ esquerda ( $r=0.986)$.

A confiabilidade intra-examinador foi excelente tanto para o dinamômetro Jamar quanto para o dinamômetro Saehan (Tabela 3).

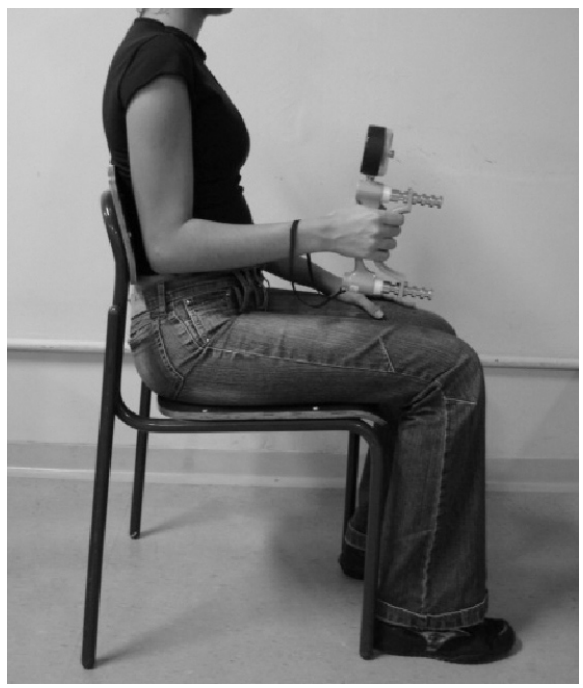

Figura 1. Posição recommendada pela ASHT (American Society of Hand). Therapists)

Tabela 1. Sequência da avaliação para os grupos de homens e mulheres pares e ímpares com os dinamômetros Jamar e Saehan

\begin{tabular}{lllll}
\hline & Mão direita & Mão esquerda & Mão direita & Mão esquerda \\
\hline GHMP & Jamar & Jamar & Saehan & Saehan \\
GHMI & Saehan & Saehan & Jamar & Jamar \\
\hline
\end{tabular}

GHMP: Grupo de homens e mulheres pares; GHMI: Grupo de homens e mulheres ímpares

Tabela 2. Valores médios da força de preensão para os dinamômetros Jamar e Saehan

\begin{tabular}{lcccccccc}
\hline & \multicolumn{4}{c}{ Mão direita } & \multicolumn{4}{c}{ Mão esquerda } \\
& \multicolumn{2}{c}{ Jamar } & \multicolumn{2}{c}{ Saehan } & \multicolumn{2}{c}{ Jamar } & \multicolumn{2}{c}{ Saehan } \\
\hline \multirow{2}{*}{ Homens $(\mathrm{n}=50)$} & 39,50 & 5,77 & 41,39 & 4,35 & 38,40 & 3,53 & 39,02 & 10,25 \\
Mulheres $(\mathrm{n}=50)$ & 22,70 & 7,42 & 24,02 & 10,72 & 21,04 & 6,48 & 21,06 & 7,19 \\
\hline
\end{tabular}

Dados são valores da média (M) e desvio padrão (DP). Valores de força de preensão manual expressos em quilograma força (kgf)

Tabela 3. Resultados do coeficiente de correlação intraclasse para os testes das mãos direita e esquerda com os dinamômetros Jamar e Saehan

\begin{tabular}{lcc}
\hline & Mão direita & Mão esquerda \\
\hline Jamar & 0,985 & 0,985 \\
Saehan & 0,981 & 0,985 \\
\hline
\end{tabular}




\section{DISCUSSÃO}

Diversas marcas de dinamômetros para a avaliação da força de preensão manual apareceram no mercado nacional nos últimos anos. Alguns exemplos são o dinamômetro TAKEY KIKI KOGIO TK 120142, o dinamômetro ENDVECO modelo $136^{43}$ e o dinamômetro SMEDLEY ${ }^{44}$. Porém, não existem estudos demonstrando a validade e a confiabilidade da maioria destes instrumentos. Frente à atual necessidade de se garantir que instrumentos de avaliação utilizados na prática clínica sejam válidos e confiáveis, é essencial que fisioterapeutas e terapeutas ocupacionais provem a validade e a confiabilidade dos seus instrumentos de avaliação ${ }^{1}$. O presente estudo fornece valores de validade e confiabilidade do dinamômetro Saehan baseados em dados derivados de testes de força de preensão manual realizados em humanos. A vantagem de se utilizar o dinamômetro Saehan é seu baixo preço comparado com o dinamômetro Jamar, o que o torna acessível aos clínicos e pesquisadores que desejarem utilizar este instrumento na prática clínica ou para o desenvolvimento de pesquisas. Além disto, o dinamômetro Jamar, considerado o "padrão ouro" para avaliar a força de preensão manual27,29, está disponível apenas no mercado internacional, não sendo mais comercializado no Brasil.

A avaliação da força de preensão manual utilizando o dinamômetro Jamar é considerada o padrão com o qual todos os outros instrumentos devem ser comparados $^{9,26-31}$. Um dos motivos para esta escolha é a alta validade e confiabilidade que este instrumento apresenta ${ }^{23-29}$. Segundo Fess $^{17}$, a confiabilidade e a validade são essenciais para determinar a efetividade de um instrumento de avaliação. A mais forte ferramenta estatística para esta análise é o $\mathrm{CCl}$, que com apenas um coeficiente, mede não apenas a associação entre duas variáveis, mas a concordância, ou seja, o grau em que os dados são os mesmos ${ }^{45}$.

Os resultados do presente estudo mostram a comparabilidade das mensurações do dinamômetro Saehan com o reconhecido dinamômetro Jamar. Os dados coletados revelaram uma excelente confiabilidade intra-examinador para os dinamômetros Jamar ( $r=0.985$ mãos direita e esquerda) e Saehan ( $r=0.981$ mão direita e $r=0.985$ mão esquerda), indicando que os resultados dos testes de força de preensão realizados com ambos os dinamômetros foram consistentes. Estudos prévios reportaram altos valores de confiabilidade intra-examinador, variando de 0.97 a 0.99 , para outros instrumentos de avaliação da força de preensão manual, incluindo os dinamômetros Baseline ${ }^{28}$, Roylan $^{27}$, Dynex ${ }^{31}$ e o BTE-Primus grip tool $^{29}$. A validade concorrente entre o dinamômetro Jamar e o dinamômetro Saehan foi excelente para os testes realizados com as mãos direita $(r=0.976) \mathrm{e}$ esquerda ( $r=0.986)$, indicando que ambos os dinamômetros medem o mesmo construto de força de preensão manual. Outros estudos utilizaram o dinamômetro Jamar como um critério padrão, comparando-o com vários instrumentos de avaliação de força de preensão manual para determinar a validade concorrente, com valores variando de 0,901 a 0,9918,23,27-29,31.

O intercâmbio entre dinamômetros para a avaliação da força de preensão manual nunca deve ser praticado. O presente estudo utilizou um dinamômetro Jamar e um dinamômetro Saehan. Não se pode afirmar que todos os dinamômetros Jamar e Saehan medem de maneira equivalente a menos que sua validade concorrente com pesos conhecidos seja demonstrada. Similarmente, devido ao fato de existir evidência de que diferentes versões do dinamômetro Jamar nem sempre medem de forma equivalente ${ }^{24}$, podese afirmar o mesmo para o dinamômetro Saehan. Assim, o mesmo dinamômetro deve ser usado para avaliar e reavaliar o paciente, quando se pretende obter medidas comparativas ${ }^{24}$.

As avaliações foram realizadas com as alças de ambos os dinamômetros reguladas na segunda posição. Estudos futuros devem determinar se testes realizados com a primeira, terceira, quarta e quinta posições da alça do dinamômetro Saehan são válidos e confiáveis quando comparados com testes realizados com as mesmas posições do dinamômetro Jamar. A coleta de dados foi realizada por apenas um avaliador; assim, nenhuma conclusão pode ser tirada com relação à confiabilidade inter-examinador do dinamômetro Saehan. Finalmente, os resultados não devem ser generalizados para crianças e idosos, pois estas faixas etárias não foram incluídas no estudo.

\section{CONCLUSÕES}

O dinamômetro Saehan hidráulico é válido, confiável e comparável com o dinamômetro Jamar hidráulico quando regulado na segunda posição, devidamente calibrado e quando instruções e posicionamento padronizados são adotados para a realização dos testes de força de preensão manual. Portanto, dados coletados com o dinamômetro Jamar são equivalentes aos dados coletados com o dinamômetro Saehan. Consequentemente, dados normativos de força de preensão manual do dinamômetro Jamar podem ser utilizados como referência para testes realizados com o dinamômetro Saehan. 


\section{REFERÊNCIAS}

1. Hammond R. Evaluation of physiotherapy by measuring outcome. Physiotherapy. 2000;86(4):170-2.

2. Bowen IJ, Sosa DM. Value of hand strenght in healthy adults. Bol Med Postgrado. 2001;17(2):57-68.

3. Incel NA, Ceceli E, Durukan PB, Erdem HR, Yorgancioglu ZR. Grip Strength: Effect of Hand Dominance. Singapore Med J. 2002;43(5):234-7.

4. Figueiredo IM, Sampaio RF, Mancini MC, Silva FCM, Souza MAP. Test of grip strength using the Jamar dynamometer. Acta Fisiatr. 2007;14(2):104-10.

5. Rauch F, Neu CM, Wassmer G, Beck B, Rieger-Wettengl G, Rietschel E, Manz F, Schoenau E. Muscle analysis by measurement of maximal isometric grip force: New reference data and clinical applications in pediatrics. Pediatr Res. 2002;51(4):505-10.

6. Moreira D, Alvarez RRA, Gogoy JR, Cambraia AN. Abordagem sobre preensão palmar utilizando o dinamômetro JAMAR®: uma revisão de literatura. $R$. Bras. Ci. e Mov. 2003;11(2):95-9.

7. Fess EE. Grip strength. In: Casanova JS. Clinical Assessment Recommendations. 2nd ed. Chicago: American Society of Hand Therapists, 1992:41-45.

8. Stephens JL, Pratt N, Michlovitz S. The reliability and validity of the Tekdyne hand dynamometer: Part II. J Hand Ther. 1996;9(1):18-26.

9. Mathiowetz V, Kashman N, Volland G, Weber K, Dowe M, Rogers, S. Grip and pinch strength: Normative data for adults. Arch Phys Med Rehabil. 1985;66(2):69-74.

10. Bechtol CO. The use of a dynamometer with adjustable handle spacings. J Bone Joint Surg. 1954;36A(4):820-4.

11. Stephens JL, Pratt N, Parks B. The reliability and validity of the Tekdyne hand dynamometer: Part I. J Hand Ther. 1996;9(1):10-17.

12. Budziareck MB, Duarte RRP, Silva MCGB. Reference values and determinants for handgrip strength in healthy subjects. Clin Nutr. 2008;27(3):357-62.

13. Günther CM, Bürger A, Rickert M, Crispin A, Schulz $\mathrm{CU}$. Grip strength in healthy caucasian adults: reference values. J Hand Surg [Am]. 2008;33(4):558-65.

14. Peolsson A, Hedlund R, Oberg B. Intra- and inter-tester reliability and reference values for hand strength. J Rehab Med. 2001;33(1):36-41.

15. Schlüssel MM, Dos Anjos LA, De Vasconcellos, MTL, Kac G. Reference values of handgrip dynamometry of healthy adults: a population-based study. Clin Nutr. 2008;27(4):601-7.

16. Fess EE. A method for checking Jamar dynamometer calibration. J Hand Ther. 1987;1:28-32.
17. Fess EE. The need for reliability and validity in hand assessment instruments. J Hand Surg [Am]. 1986;11(5):621-3.

18. Beaton DE, O'driscoll SW, Richards RR. Grip strength testing using the BTE work simulator and the Jamar dynamometer: a comparative study. J Hand Surg [Am]. 1995;20(2):293-8.

19. Clerke A, Clerke J. A literature review of the effect of handedness on isometric grip strength: differences of the left and right hands. Am J Occup Ther. 2001;55(2):20611.

20. Taylor C, Shechtman O. The use of the rapid exchange grip test in detecting sincerity of effort, Part I: administration of the test. J Hand Ther. 2000;13(3):195202.

21. Bear-Lehman J, Abreu BC. Evaluating the hand: issues in reliability and validity. Phys Ther. 1989;69(12):1025-33.

22. Jones LA. The assessment of hand function: a critical review of techniques. J Hand Surg [Am]. 1989;14(2):221-8.

23. Bellace JV, Healy D, Besser MP, Byron T, Hohman L. Validity of the Dexter Evaluation System's Jamar dynamometer attachment for assessment of hand grip strength in a normal population. J Hand Ther. 2000;13(1):46-51.

24. Flood-Joy M, Mathiowetz V. Grip strength measurement: a comparison of two instruments. Occup Ther J Res. 1987;7:235-43.

25. Hamilton A, Balnave R, Adams R. Grip strength testing reliability. J Hand Ther. 1994;7:163-70.

26. Hamilton GF, Mcdonald C, Chenier TC. Measurement of grip strength - validity and reliability of the sphygmomanometer and Jamar grip dynamometer. J Orthop Sports Phys Ther. 1992;16:215-9.

27. Mathiowetz V. Comparison of Rolyan and Jamar dynamometers for measuring grip strength. Occup Ther Int. 2002;9(3):201-9.

28. Mathiowetz V, Vizenor L, Melander D. Comparison of baseline instruments to the Jamar dynamometer and the $B \& L$ engineering pinch gauge. Occup Ther J Res. 2000;20:147-62.

29. Shechtman O, Davenport R, Malcolm M, Nabavi D. Reliability and validity of the BTE-Primus grip tool. J Hand Ther. 2003;16(1):36-42.

30. Brown A, Cramer LD, Eckhaus D, Schmidt J, Ware L, Mackenzie E. Validity and reliability of the dexter hand evaluation and therapy system in hand-injured patients. J Hand Ther. 2000;13(1):37-45. 


\section{Referências (cont.)}

31. Shechtman O, Gestewitz L, Kimble C. Reliability and Validity of the DynEx Dynamometer. J Hand Ther. 2005; 18:339-47.

32. Portney LG, Watkins MP. Foundations of Clinical Research: Applications to Practice. 2nd ed. Norwalk, CT: Appleton \& Lange; 2000.

33. Häkkinen A, Rinne M, Vasankari T, Santtila M, Häkkinen K, Kyröläinen H. Association of physical fitness with health-related quality of life in Finnish young men. Health Qual Life Outcomes. 2010; 29;8:15.

34. Fairhall N, Aggar C, Kurrle SE, Sherrington C, Lord S, Lockwood K, Monaghan N, Cameron ID. Frailty Intervention Trial (FIT). BMC Geriatr. 2008;.13;8:27.

35. Ng GYF, Fan ACC. Does elbow position affect strength and reproducibility of power grip measurements? Physiotherapy. 2001;87(2):68-72.

36. Oxford KL. Elbow positioning for maximum grip performance. J Hand Ther. 2000;13(1):33-6.

37. Richards LG. Posture effects on grip strength. Arch Phys Med Rehabil. 1997;78(10):1154-6.

38. Richards LG, Olson B, Palmiter TP. How forearm position affects grip strength. Am J Occup Ther. 1996;50(2):133-8.

39. Mcgarvey SR, Morrey BF, Askew LJ, An KN. Reliability of isometric strength testing: temporal factors and strength variation. Clin Orthop Res. 1984;185:301-5.
40.Johansson CA, Kent BE, Shepard KF. Relationship between verbal command volume and magnitude of muscle contraction. Phys Ther. 1983;63(8):1260-5.

41. Stratford PW. Summarizing the results of multiple strength trials: truth or consequence. Physio Can. 1992;44:14-8.

42. Barbosa AR, Souza JMP, Lebrão ML, Laurenti R, Marucci MFN. Functional limitations of Brazilian elderly by age and gender differences: data from SABE Survey. Caderno de Saúde Pública. 2005;21(4):1177-85.

43. Esteves AC, Reis DC, Caldeira RM, Leite RM, Moro ARP, Borges Jr, NG. Força de preensão, lateralidade, sexo e características antropométricas da mão de crianças em idade escolar. Revista Brasileira de Cineantropometria \& Desempenho Humano. 2005;7(2): 69-75.

44. Frederiksen H, Hjelmborg J, Jakob Mortensen Mcgue M.; Vaupel JW, Christensen K. Age Trajectories of Grip Strength: Cross-sectional and Longitudinal Data Among 8,342 Danes Aged 46 to 102. Ann Epidemiol. 2006;16(7):554-62.

45. Kramer MS, Feinstein AR. Clinical biostatistics: LIV. The biostatistics of concordance. Clin Pharmacol Ther. 1981;29:111-23. 